\title{
Effect of blood sampling schedule and method of calculating the area under the curve on validity and precision of glycaemic index values
}

\author{
Thomas M. S. Wolever \\ Department of Nutritional Sciences, University of Toronto, and Glycaemic Index Testing, Inc., Toronto, Ontario, \\ Canada
}

(Received 25 March 2003 - Revised 26 September 2003 - Accepted 3 November 2003)

\begin{abstract}
To evaluate the suitability for glycaemic index (GI) calculations of using blood sampling schedules and methods of calculating area under the curve (AUC) different from those recommended, the GI values of five foods were determined by recommended methods (capillary blood glucose measured seven times over $2.0 \mathrm{~h}$ ) in forty-seven normal subjects and different calculations performed on the same data set. The AUC was calculated in four ways: incremental AUC (iAUC; recommended method), iAUC above the minimum blood glucose value (AUCmin), net AUC (netAUC) and iAUC including area only before the glycaemic response curve cuts the baseline (AUCcut). In addition, iAUC was calculated using four different sets of less than seven blood samples. GI values were derived using each AUC calculation. The mean GI values of the foods varied significantly according to the method of calculating GI. The standard deviation of GI values calculating using iAUC (20.4), was lower than six of the seven other methods, and significantly less $(P<0 \cdot 05)$ than that using netAUC (24.0). To be a valid index of food glycaemic response independent of subject characteristics, GI values in subjects should not be related to their AUC after oral glucose. However, calculating GI using AUCmin or less than seven blood samples resulted in significant $(P<0.05)$ relationships between GI and mean AUC. It is concluded that, in subjects without diabetes, the recommended blood sampling schedule and method of AUC calculation yields more valid and/or more precise GI values than the seven other methods tested here. The only method whose results agreed reasonably well with the recommended method (ie. within $\pm 5 \%$ ) was AUCcut.
\end{abstract}

Glycaemic index: Blood glucose response: Methodology: Dietary carbohydrate

The glycaemic index (GI) is a classification of carbohydrate foods based on their acute blood glucose responses; it should not be used without also considering information about the chemical composition of foods (Jenkins et al. 1981). The GI has been recommended to help guide food choice (Food and Agriculture Organization, 1998), because low-GI foods have been shown to improve blood glucose control in people with diabetes (Brand-Miller et al. 2003), to increase insulin sensitivity (Frost et al. 1998) and $\beta$-cell function (Wolever \& Mehling, 2002), and to reduce serum triacylglycerol (Jenkins et al. 1987). In addition, a low-GI diet has been associated with reduced risk for developing diabetes and CVD in some studies (Salmeron et al. 1997a,b; Liu et al. 2000), but not all (Meyer et al. 2000; Van Dam et al. 2000). Widespread use of the GI, as recommended, requires a standardized method for determining the GI of foods that is valid and precise.

The GI is defined as the incremental area under the blood glucose response curve (AUC) after consumption of a $50 \mathrm{~g}$ available-carbohydrate portion of a food expressed as a percentage of that after $50 \mathrm{~g}$ oral glucose.
For subjects without diabetes, the Food and Agriculture Organization (1998) recommends determining GI by collecting seven blood samples over $2 \mathrm{~h}$ and calculating incremental AUC (iAUC; Jenkins et al. 1981; Wolever \& Jenkins, 1986). Shorter tests and less frequent blood sampling would reduce costs; other methods of calculating AUC have been used (Wolever, 1989; Ha et al. 1992), but the effect of these variables on the GI is not known. Thus, the purpose of the present paper was to compare the suitability for GI calculations of using different blood sampling schedules and other ways of calculating AUC than those recommended by the Food and Agriculture Organization (1998).

\begin{abstract}
Methods
Data from a multicentre trial were used, the methods and results of which have been published by Wolever et al. (2003). The GI values of five foods were determined in subjects without diabetes in seven centres using the method recommended by the Food and Agriculture Organization (1998). Results from the five centres that measured
\end{abstract}


glucose in whole blood or plasma obtained from capillary blood samples were used in this analysis. Results from the two centres that measured glucose in venous plasma were not used because of higher variability of glycaemic responses and GI values (Wolever et al. 2003). In brief, subjects without diabetes (twenty-three male, twenty-four female; age 28.9 (SEM 1.2) (range 19-50) years, BMI 23.3 (SEM 0.5) (range 16.8-35.0) $\mathrm{kg} / \mathrm{m}^{2}$ ) were studied on eight occasions in the morning after a $10-14 \mathrm{~h}$ overnight fast. On each occasion, after a fasting blood sample was obtained, subjects consumed a test meal containing $50 \mathrm{~g}$ available carbohydrate (defined as total carbohydrate minus dietary fibre), with further blood samples being taken $15,30,45,60,90$ and $120 \mathrm{~min}$ after starting to eat. On three occasions (first, middle and last trials), subjects consumed $50 \mathrm{~g}$ anhydrous glucose. On the other occasions subjects consumed instant potato, white bread, polished rice, white spaghetti or pearled barley in randomized order. The AUC was calculated for each trial and the AUC after consuming each food was expressed as a percentage of the average AUC after oral glucose taken by the same subject. The average of these values for each food was the GI of the food.

For this analysis, eight different calculations were performed on the same data set and the results compared. Table 1 shows the results of AUC calculations using the different methods on real sets of blood glucose profiles from one subject. The recommended calculation used all seven blood samples with iAUC being calculated. The iAUC includes all area below the curve and above the fasting concentration, with any area beneath fasting being ignored (Wolever \& Jenkins, 1986). The iAUC was also calculated on four different combinations of four, five or six blood samples representing a shorter test period, less frequent blood sampling or both. Net incremental AUC (netAUC) (Gannon et al. 1989) includes all incremental area below the curve, including the area below the fasting concentration. Since it is calculated by applying the trapezoid rule to both positive and negative blood glucose increments, the effect is to subtract the area below the fasting level from that above. AUCcut (Ha et al. 1992) is calculated in the same way as iAUC, but only includes the area before the blood glucose concentration drops below (cuts) the baseline (fasting concentration); the area after the glucose concentration cuts the baseline is not included. The iAUC above the lowest blood glucose concentration attained (AUCmin) (Vorster et al. 1990) is calculated by subtracting the lowest blood glucose concentration attained during the test period from each of the other blood glucose concentrations, and calculating the AUC by applying the trapezoid rule to the resulting increments.

There is no easy way to measure the validity of different methods of calculating the GI. The approach taken here was to determine the correlation between the mean GI (average GI of the five foods) and the mean AUC after oral glucose (average of three trials) for the forty-seven subjects. The rationale for this approach is that the GI is intended to indicate the blood-glucose-raising potential of foods independent of the glycaemic response of the subject. Thus, for valid methods of calculating GI, there should be no correlation between the GI values obtained and the AUC after oral glucose. The presence of a significant correlation between GI and AUC would indicate that the method was not valid, because the GI values obtained depended on the glucose tolerance status of the subject.

The standard deviation of the mean GI values is a measure of precision, which is the degree of variation of values about their mean value. Since the standard deviation

Table 1. Sample calculations (using the same data from one subject for each method)*

\begin{tabular}{|c|c|c|c|c|c|c|c|c|c|c|}
\hline \multirow[b]{2}{*}{ Time $(\min ) \ldots$} & & \multicolumn{7}{|c|}{ Blood glucose concentrations (mmol/l) } & \multirow[b]{2}{*}{ AUC } & \multirow[b]{2}{*}{ GI } \\
\hline & & 0 & 15 & 30 & 45 & 60 & 90 & 120 & & \\
\hline \multicolumn{11}{|l|}{ Method } \\
\hline \multirow[t]{2}{*}{ iAUC† } & Glucose & $3 \cdot 67$ & $6 \cdot 11$ & 6.06 & 4.44 & $3 \cdot 17$ & $3 \cdot 61$ & 4.00 & 85.8 & \\
\hline & Food & 3.94 & 5.00 & $5 \cdot 11$ & 3.44 & 3.50 & 3.83 & 4.33 & 35.4 & 41 \\
\hline \multirow[t]{2}{*}{$\mathrm{AUC}_{1}$} & Glucose & 3.67 & $6 \cdot 11$ & 6.06 & 4.44 & 3.17 & $3 \cdot 61$ & - & $81 \cdot 7$ & \\
\hline & Food & 3.94 & 5.00 & $5 \cdot 11$ & 3.44 & 3.50 & 3.83 & - & 30.8 & 38 \\
\hline \multirow{2}{*}{$\mathrm{AUC}_{2}$} & Glucose & 3.67 & $6 \cdot 11$ & 6.06 & 4.44 & 3.17 & - & - & 81.7 & \\
\hline & Food & 3.94 & 5.00 & $5 \cdot 11$ & 3.44 & 3.50 & - & - & 30.8 & 38 \\
\hline \multirow[t]{2}{*}{$\mathrm{AUC}_{3}$} & Glucose & 3.67 & - & 6.06 & - & $3 \cdot 17$ & $3 \cdot 61$ & 4.00 & 69.7 & \\
\hline & Food & 3.94 & - & $5 \cdot 11$ & - & 3.50 & 3.83 & 4.33 & 34.9 & 50 \\
\hline \multirow[t]{2}{*}{$\mathrm{AUC}_{4}$} & Glucose & 3.67 & - & 6.06 & 4.44 & - & $3 \cdot 61$ & - & 75.6 & \\
\hline & Food & 3.94 & - & $5 \cdot 11$ & 3.44 & - & 3.83 & - & 23.7 & 31 \\
\hline \multirow[t]{2}{*}{ netAUC } & Glucose & 3.67 & $6 \cdot 11$ & 6.06 & 4.44 & 3.17 & $3 \cdot 61$ & 4.00 & 75.9 & \\
\hline & Food & 3.94 & 5.00 & $5 \cdot 11$ & 3.44 & 3.50 & 3.83 & 4.33 & $18 \cdot 6$ & 25 \\
\hline \multirow[t]{2}{*}{ AUCcut } & Glucose & 3.67 & $6 \cdot 11$ & 6.06 & 4.44 & $3 \cdot 17$ & $3 \cdot 61$ & 4.00 & $81 \cdot 7$ & \\
\hline & Food & 3.94 & 5.00 & $5 \cdot 11$ & 3.44 & 3.50 & 3.83 & 4.33 & $30 \cdot 8$ & 38 \\
\hline \multirow[t]{2}{*}{ AUCmin } & Glucose & 3.67 & $6 \cdot 11$ & 6.06 & 4.44 & $3 \cdot 17$ & $3 \cdot 61$ & 4.00 & 135.9 & \\
\hline & Food & 3.94 & 5.00 & $5 \cdot 11$ & 3.44 & 3.50 & 3.83 & 4.33 & 78.6 & 58 \\
\hline
\end{tabular}

AUC, area under the curve; GI, glycaemic index; iAUC, incremental area under the curve; $\mathrm{AUC}_{1}$, incremental area under the curve using $0,15,30,45,60$ and 90 min glucose values; $A \mathrm{UC}_{2}$, incremental area under the curve using $0,15,30,45$ and 60 min glucose values; $\mathrm{AUC}_{3}$, incremental area under the curve using $0,30,60,90$ and 120 min glucose values; $A U C_{4}$, incremental area under the curve using $0,30,45$ and 90 min glucose values; netAUC, net incremental area under the curve; AUCcut, incremental area under the curve up to first cut of baseline; AUCmin, incremental area under the curve above minimum glucose.

${ }^{*}$ For details of procedures and calculations, see pp. 296-297.

† Recommended method (Food and Agriculture Organization, 1998). 
of the GI is largely determined by within-subject variation (Wolever, 1992), the CV $(\mathrm{CV}=100 \times \mathrm{sD} /$ mean value $)$ of the AUC after the repeated trials of oral glucose was calculated.

The mean GI values for each of the five foods from each of the five centres calculated using each of the different methods were compared with those calculated using the recommended method using the Bland \& Altman (1986) procedure. They defined the 'limits of agreement' as being the mean value $\pm 1.96 \times \mathrm{SD}$ of the differences between the results from two methods, representing, therefore, the range within which $95 \%$ of the differences between the methods would be expected to fall. In the present study, the analysis involved comparison of twenty-five values (five foods and five centres) for each method $v$. the recommended method. Using the recommended method, foods were classified as high $(>69)$, medium $(56-69)$ or low $(<56)$ GI (Brand-Miller et al. 2002), and the number of misclassifications for each alternate method was calculated.

Unless otherwise indicated, results are expressed as mean values with their standard errors. The mean values, standard deviations and CV of the AUC values after the repeated trials of oral glucose taken by each subject were subjected to repeated-measures ANOVA examining for differences between subjects and methods of calculating AUC. Mean values and standard deviations of the GI values were subjected to ANOVA examining for the main effects of food, method of calculating AUC (method), subject, and the food $\times$ method interaction. If significant effects were found by ANOVA, the significance of differences between individual mean values was determined using the Newman-Keuls method to adjust for multiple comparisons (Snedecor \& Cochran, 1980). Pearson's correlation coefficients between AUC and GI were calculated by the method of least squares (Lotus 123, 1997 edition; Lotus Development Corp., Cambridge, MA, USA). Effects were considered to be statistically significant if two-tailed $P<0 \cdot 05$.

\section{Results}

The mean blood glucose responses after oral glucose and the five foods are shown in Fig. 1. The different methods

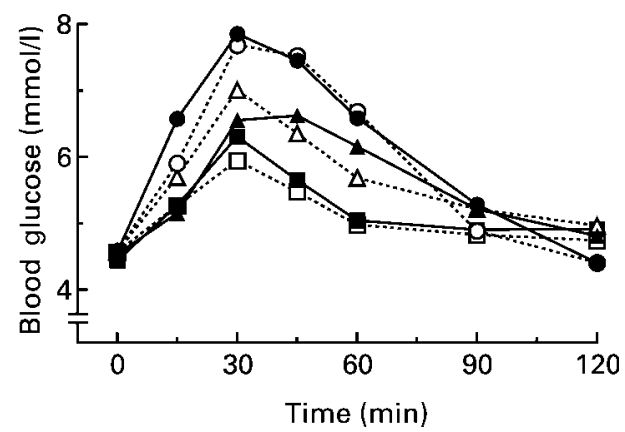

Fig. 1. Blood glucose concentrations of forty-seven subjects after consuming $50 \mathrm{~g}$ available carbohydrate from glucose $(\bullet)$, instant potato $(\bigcirc)$, white bread $(\boldsymbol{\Delta})$, polished rice $(\triangle)$, white spaghetti $(\boldsymbol{\square})$ and pearled barley $(\square)$. Values are means. For details of subjects and procedures, see pp. 296-297. of calculating iAUC yielded significantly different mean values. After oral glucose (Table 2), AUCmin was significantly greater $(P<0.05)$ than iAUC and AUCcut, which in turn were significantly greater $(P<0.05)$ than $\mathrm{AUC}_{1}$ and $\mathrm{AUC}_{4}$, which in turn were significantly greater $(P<0 \cdot 05)$ than $\mathrm{AUC}_{2}$. Mean values for netAUC and $\mathrm{AUC}_{3}$ were intermediate between iAUC and $\mathrm{AUC}_{1}$. Statistical analysis of the AUC values after the five foods revealed significant main effects of food $\left(F_{4,28} 240 \cdot 9, P<0.001\right)$ and method of AUC calculation $\left(F_{7,28} 26 \cdot 5, P<0.001\right)$, and a significant food $\times$ method interaction $\left(F_{28,1794} 1.87, P=0.003\right)$. The significant food $\times$ method interaction indicates that the difference in AUC between foods differed significantly depending on the method of calculating AUC. The greatest AUC after the five foods was obtained with AUCmin and the smallest with $\mathrm{AUC}_{2}$, with the others being intermediate (Table 3).

The degree of within-subject variation, assessed by standard deviations and $\mathrm{CV}$ of the repeated glucose trials, varied significantly by method of AUC calculation (Table 2). The largest $\mathrm{CV}$ was obtained with netAUC $(28.0 \%)$ and the smallest with $\mathrm{AUC}_{2}(20.7 \%)$ and AUCmin $(20.5 \%)$, with the $\mathrm{CV}$ of iAUC, the recommended AUC calculation method, being intermediate $(23.4 \%)$.

Statistical analysis of the GI values calculated using the different AUC methods revealed significant main effects of food $\left(F_{4,28} 649.9, P<0.001\right)$ and method of AUC calculation $\left(F_{7,28} 6 \cdot 86, P<0 \cdot 001\right)$, but the food $\times$ method interaction was not significant $\left(F_{28,1794} 0 \cdot 86, P=0 \cdot 68\right)$. When individual mean values were compared, mean GI calculated using AUCmin, 57.2, was significantly less than that calculated using the other methods (61.4-66.4), which in turn, did not differ significantly from each other (Table 3). The method of calculating AUC significantly

Table 2. Areas under the curves calculated by different methods after repeated trials of oral glucose in forty-seven subjects*

(Mean values, standard deviations and coefficients of variation)

\begin{tabular}{|c|c|c|c|c|c|c|}
\hline \multirow[b]{2}{*}{ Method } & \multicolumn{2}{|c|}{$\begin{array}{c}\text { Mean } \\
(\mathrm{mmol} \times \min / \mathrm{l})\end{array}$} & \multicolumn{2}{|c|}{$\begin{array}{c}\mathrm{SD} \\
(\mathrm{mmol} \times \mathrm{min} / \mathrm{l})\end{array}$} & \multicolumn{2}{|c|}{ CV (\%) } \\
\hline & Mean & SD & Mean & SD & Mean & SD \\
\hline ¡AUC† & $196^{b}$ & 11 & $45 \cdot 2^{a b c}$ & $4 \cdot 7$ & $23 \cdot 4^{\mathrm{abc}}$ & $2 \cdot 1$ \\
\hline $\mathrm{AUC}_{1}$ & $179^{c}$ & 9 & $39 \cdot 4^{c}$ & 3.9 & $22 \cdot 4^{\mathrm{bc}}$ & $2 \cdot 0$ \\
\hline $\mathrm{AUC}_{2}$ & $138^{d}$ & 6 & $26 \cdot 6^{d}$ & $2 \cdot 2$ & $20 \cdot 7^{c}$ & $2 \cdot 0$ \\
\hline $\mathrm{AUC}_{3}$ & $187^{\mathrm{bc}}$ & 11 & $47 \cdot 4^{\mathrm{abc}}$ & 4.6 & $26 \cdot 1^{a b}$ & $2 \cdot 2$ \\
\hline $\mathrm{AUC}_{4}$ & $177^{\mathrm{C}}$ & 9 & $41 \cdot 9^{b c}$ & 3.9 & $25 \cdot 3^{\mathrm{ab}}$ & $2 \cdot 3$ \\
\hline netAUC & $184^{\mathrm{bc}}$ & 11 & $49 \cdot 6^{\mathrm{ab}}$ & $5 \cdot 0$ & $28 \cdot 0^{a}$ & $2 \cdot 6$ \\
\hline AUCcut & $195^{b}$ & 11 & $46 \cdot 4^{\mathrm{abc}}$ & $4 \cdot 8$ & $24 \cdot 3^{a b c}$ & $2 \cdot 2$ \\
\hline AUCmin & $258^{a}$ & 12 & $52 \cdot 1^{a b c}$ & 5.5 & $20 \cdot 5^{c}$ & $2 \cdot 0$ \\
\hline$P \ddagger$ & \multicolumn{2}{|c|}{$<0.001$} & \multicolumn{2}{|c|}{$<0.001$} & \multicolumn{2}{|c|}{$<0.001$} \\
\hline
\end{tabular}

iAUC, incremental area under the curve, $\mathrm{AUC}_{1}$, iAUC using 0, 15, 30, 45, 60 and $90 \mathrm{~min}$ glucose values; $\mathrm{AUC}_{2}$, iAUC using $0,15,30,45$ and $60 \mathrm{~min}$ glucose values; $\mathrm{AUC}_{3}$, iAUC using $0,30,60,90$ and $120 \mathrm{~min}$ glucose values; $\mathrm{AUC}_{4}$, iAUC using $0,30,45$ and $90 \mathrm{~min}$ glucose values; netAUC, net incremental area under the curve; AUCmin, incremental AUC above minimum glucose.

${ }^{a b c d}$ Mean values with unlike superscript letters were significantly different $(P<0.05)$.

* For details of procedures and calculations, see pp. 296-297.

† Recommended method (Food and Agriculture Organization, 1998).

$\ddagger$ Significance of main effect of method of calculation from ANOVA. 
Table 3. Area under the curve $(\mathrm{mmol} \times \mathrm{min} / \mathrm{l})$ and glycaemic index values for each food calculated using different methods*

\begin{tabular}{|c|c|c|c|c|c|c|c|c|c|c|c|c|}
\hline \multirow[b]{2}{*}{ Method } & \multicolumn{2}{|c|}{ Potato } & \multicolumn{2}{|c|}{ Bread } & \multicolumn{2}{|c|}{ Rice } & \multicolumn{2}{|c|}{ Spaghetti } & \multicolumn{2}{|c|}{ Barley } & \multicolumn{2}{|c|}{ Mean of } \\
\hline & Mean & SD & Mean & SD & Mean & SD & Mean & SD & Mean & SD & Means & SD \\
\hline iAUC† & 176 & 77 & 134 & 57 & 135 & 76 & 94 & 56 & 69 & 46 & $122^{b}$ & $62^{a}$ \\
\hline $\mathrm{AUC}_{1}$ & 165 & 65 & 118 & 43 & 117 & 62 & 80 & 45 & 61 & 35 & $108^{b}$ & $50^{b}$ \\
\hline $\mathrm{AUC}_{2}$ & 127 & 40 & 83 & 26 & 89 & 37 & 63 & 33 & 49 & 22 & $82^{\mathrm{C}}$ & $32^{c}$ \\
\hline $\mathrm{AUC}_{3}$ & 174 & 78 & 136 & 57 & 136 & 77 & 96 & 56 & 69 & 45 & $122^{\mathrm{b}}$ & $63^{a}$ \\
\hline netAUC & 165 & 84 & 132 & 58 & 130 & 79 & 93 & 57 & 65 & 49 & $117^{\mathrm{b}}$ & $65^{a}$ \\
\hline AUCcut & 176 & 77 & 134 & 57 & 133 & 77 & 92 & 58 & 69 & 47 & $121^{b}$ & $63^{a}$ \\
\hline AUCmin & 221 & 81 & 151 & 58 & 154 & 71 & 102 & 53 & 85 & 44 & $143^{a}$ & $61^{a}$ \\
\hline \multicolumn{13}{|c|}{ Glycaemic index } \\
\hline IAUC† & 91.5 & $25 \cdot 1$ & $71 \cdot 0$ & $20 \cdot 7$ & $68 \cdot 7$ & $22 \cdot 6$ & $47 \cdot 6$ & $18 \cdot 1$ & $35 \cdot 2$ & $15 \cdot 7$ & $62 \cdot 8^{a}$ & $20 \cdot 4^{a b}$ \\
\hline $\mathrm{AUC}_{1}$ & 94.0 & $25 \cdot 1$ & $68 \cdot 1$ & $19 \cdot 0$ & 65.6 & $23 \cdot 2$ & $44 \cdot 6$ & $19 \cdot 3$ & 34.7 & $15 \cdot 2$ & $61 \cdot 4^{a}$ & $20 \cdot 3^{b}$ \\
\hline $\mathrm{AUC}_{2}$ & 94.8 & $23 \cdot 5$ & 63.4 & $19 \cdot 5$ & $67 \cdot 1$ & 24.4 & $47 \cdot 6$ & $23 \cdot 8$ & $37 \cdot 6$ & $17 \cdot 1$ & $62 \cdot 1^{\mathrm{a}}$ & $21 \cdot 7^{a b}$ \\
\hline $\mathrm{AUC}_{3}$ & 94.9 & $27 \cdot 0$ & $76 \cdot 9$ & 23.7 & $72 \cdot 1$ & $22 \cdot 5$ & $51 \cdot 7$ & $20 \cdot 1$ & $36 \cdot 4$ & $16 \cdot 5$ & $66 \cdot 4^{\mathrm{a}}$ & $21.9^{a b}$ \\
\hline netAUC & 90.9 & 30.7 & $75 \cdot 4$ & $23 \cdot 6$ & $71 \cdot 0$ & 28.0 & $50 \cdot 0$ & $19 \cdot 6$ & $34 \cdot 1$ & $17 \cdot 9$ & $64 \cdot 3^{a}$ & $24 \cdot 0^{a}$ \\
\hline AUCcut & $92 \cdot 1$ & $25 \cdot 3$ & $71 \cdot 3$ & 21.5 & $67 \cdot 6$ & $23 \cdot 8$ & $46 \cdot 1$ & $19 \cdot 2$ & 34.9 & $16 \cdot 5$ & $62 \cdot 4^{\mathrm{a}}$ & $21 \cdot 2^{a b}$ \\
\hline AUCmin & $86 \cdot 8$ & $22 \cdot 7$ & $60 \cdot 8$ & $20 \cdot 2$ & 62.4 & 24.6 & $41 \cdot 2$ & $19 \cdot 2$ & 34.9 & $16 \cdot 0$ & $57 \cdot 2^{b}$ & $20 \cdot 6^{a b}$ \\
\hline
\end{tabular}

iAUC, incremental area under the curve; $\mathrm{AUC}_{1}$, incremental area under the curve using 0, 15, 30, 45, 60 and 90 min glucose values; $A \cup C_{2}$, incremental area under the curve using $0,15,30,45$ and 60 min glucose values; $A_{U C}$, incremental area under the curve using $0,30,60$, 90 and 120 min glucose values; $A \cup C_{4}$, incremental area under the curve using 0, 30, 45 and 90 min glucose values; netAUC, net incremental area under the curve; AUCcut, incremental area under the curve up to first cut of baseline; AUCmin, incremental area under the curve above minimum glucose.

${ }^{a b c}$ Mean values with unlike superscript letters were significantly different $(P<0.05)$.

${ }^{*}$ For details of procedures and calculations, see pp. 296-297.

† Recommended method (Food and Agriculture Organization, 1998).

affected not only the mean GI values, but also the standard deviation of the GI values $\left(F_{7,28} 3 \cdot 13, P=0 \cdot 014\right.$; Table 3$)$. The mean standard deviation obtained using $\mathrm{AUC}_{1}, 20 \cdot 3$, was significantly less $(P<0 \cdot 05)$ than that using netAUC (24.0). The recommended method of calculating AUC, iAUC, had the second lowest mean standard deviation (20.4). Although by the primary statistical analysis mean standard deviation of GI values obtained using AUCcut (21.2), did not differ significantly from that using iAUC, the standard deviation of the GI value for each of the five foods was greater with AUCcut than iAUC, a difference which, though small, was significant by paired $t$ test (0.82 (SD 0.17), $P<0 \cdot 01$ ).

The correlation between AUC and GI was statistically significant for $\mathrm{AUC}_{2}(r-0.506, \quad P<0.001), \quad \mathrm{AUC}_{4}$ $(r-0.308, \quad P=0.035)$ and AUCmin $(r-0.430$, $P=0.003$ ), with those for $\mathrm{AUC}_{1}$ and $\mathrm{AUC}_{3},-0.23$ and -0.21 respectively approaching significance $(P=0.12$ and $P=0.16$ respectively). The correlations between AUC and GI were not statistically significant for iAUC $(r-0.139, P=0.35)$, netAUC $(r \quad 0.140, P=0.35)$ and AUCcut $(r-0.112, P=0.45)$.

The limits of agreement between each method and the recommended method expressed in absolute terms (with $\%$ mean GI value calculated using the recommended method in parentheses) were as follows: $\mathrm{AUC}_{1}$ 7.0 (11.2), $\mathrm{AUC}_{2} 13.5$ (21.4), $\mathrm{AUC}_{3} 6.6$ (10.5), $\mathrm{AUC}_{4} 7.6$ (12.1), netAUC $8.9(14 \cdot 2)$, AUCmin 13.0 (20.7), AUCcut $3 \cdot 3(5 \cdot 1)$. The limits of agreement for the different methods were directly correlated $(r 0.966, P<0.001)$ with the number of foods misclassified using the different methods (n): $\mathrm{AUC}_{1} 2(8 \%), \mathrm{AUC}_{2} 4(16 \%), \mathrm{AUC}_{3} 2(8 \%), \mathrm{AUC}_{4} 2$ $(8 \%)$, netAUC $3(12 \%)$, AUCmin $7(28 \%)$, AUCcut 2 $(8 \%)$.

\section{Discussion}

The present results suggest that the method recommended by the Food and Agriculture Organization (1998), based on taking seven blood samples over $2.0 \mathrm{~h}$ and calculating iAUC, is the best method for calculating GI of those foods tested here. Other methods resulted in GI values that either were correlated with the glucose tolerance status of the different subjects or were more variable than the recommended method. Most of the methods yielded GI values that did not agree well with the recommended method, with limits of agreement ranging from \pm 10.5 to $\pm 21.4 \%$. Only one of the alternative methods, AUCcut, yielded mean GI values $95 \%$ of which were within $\pm 5 \%$ of the recommended method.

There is no universally 'right' or 'wrong' way to measure blood glucose responses. Different methods are required for different purposes. For example, to determine whether a new treatment for diabetes reduces blood glucose concentrations, total AUC may be preferred over iAUC, since the former is a measure of the average blood glucose concentration over time, whereas the latter is a measure of change over time. Calculating netAUC may be more appropriate for measuring postprandial responses of variables such as NEFA, whose concentrations normally fall after eating. This is because netAUC can have a negative value, whereas the minimum value of iAUC is 0 .

The GI is intended to be an index of the relative bloodglucose-raising potential of the available carbohydrate in different foods. For this concept to be valid and useful, the GI value of the same food must be the same in different subjects. Glycaemic responses vary from day-to-day within subjects, and also vary between subjects. To distinguish 
between these sources of variation, repeated trials of both the reference and test food must be performed. When blood glucose response trials of two test foods and one reference food were repeated four times in each of twelve heterogeneous subjects with diabetes, there was no significant difference in GI between subjects, despite 4-fold variation in mean iAUC values between subjects (Wolever et al. 1990). This is consistent with the present results showing no significant correlation between iAUC and GI. The GI is a ratio of two independently variable values, and the statistical problems associated with the use of ratios have been described by Allison et al. (1995). The presence of a significant correlation between a ratio and its denominator indicates that the ratio does not adequately control for the denominator (Allison et al. 1995). Thus, methods of AUC calculation resulting in a significant correlation between GI and AUC cannot be considered valid for determining the GI of foods.

Ha et al. (1992) studied the glycaemic responses elicited by three different fruits taken by a group of fifteen subjects with impaired glucose tolerance or diabetes and compared the results after calculating the AUC in six different ways. They concluded that iAUC and AUCcut were more appropriate than netAUC, because netAUC resulted in larger standard deviations, and also that less frequent blood sampling increased the standard deviation of glycaemic responses. The results of Ha et al. (1992) cannot be compared exactly with the present results because the standard deviations calculated by Ha et al. (1992) included both within- and between-subject variation, and no results for GI were presented. Nevertheless, the present results also show that calculating netAUC, and reducing the frequency of blood sampling, tended to result not only in higher within-subject variation of glycaemic responses than other methods, but also higher standard deviations of the GI values.

AUC methods iAUC, $\mathrm{AUC}_{1}$ and $\mathrm{AUC}_{2}$ represent the same frequency of blood sampling, but a shorter test period; $2.0 \mathrm{~h}$ for iAUC, $1.5 \mathrm{~h}$ for $\mathrm{AUC}_{1}$ and $1.0 \mathrm{~h}$ for $\mathrm{AUC}_{2}$. It is of interest that as the time over which blood glucose was measured decreased, within-subject variation tended to decrease from $23.4 \%$ for $2.0 \mathrm{~h}$ to $20.7 \%$ for $1.0 \mathrm{~h}$ (Table 2). This suggests that, in normal subjects, blood glucose concentrations during the second postprandial hour are more variable within-subjects from day-today than those during the first hour. Although reducing within-subject variation is desirable for the most precise GI results, reducing the time over which blood samples were taken was also associated with a progressively higher correlation between GI and AUC, a factor that tends to make the GI values invalid.

It is not possible to say from the present results what would happen to GI values if the test was carried out for more than $2.0 \mathrm{~h}$. Using tests conducted over $5 \mathrm{~h}$ in normal and diabetic subjects, Gannon \& Nuttal (1987) showed that the AUC value and the relative glucose area of foods could be markedly affected by the time over which the test was done. As the time of blood sampling increased from 1 to $5 \mathrm{~h}$, AUC values after oral glucose decreased by $>50 \%$, relative glucose area values for legumes increased by approximately $100 \%$, relative glucose area values for sucrose, fructose and milk decreased by $>50 \%$ and relative glucose area values for potatoes, bread, oats and rice did not change very much. This does not agree with the present results, which showed that as the time of blood sampling increased from $1.0 \mathrm{~h}\left(\mathrm{AUC}_{2}\right)$ to $2.0 \mathrm{~h}$ (iAUC), AUC increased significantly by approximately $40 \%$, but there was no effect on mean GI. The lack of agreement is because of the different methods used to calculate the AUC. Gannon \& Nuttall (1987) used netAUC, in which area below the baseline is subtracted from that above. Since after oral glucose blood glucose tends to undershoot the baseline, the longer the time over which blood glucose is measured, the more time the blood glucose is below baseline, the more area below the baseline there is to subtract, and the lower the netAUC. On the other hand, iAUC cannot decrease as the time of the test is extended, because area beneath the baseline is ignored.

It is concluded that the blood sampling schedule and method of AUC calculation recommended by the Food and Agriculture Organization (1998) for subjects without diabetes results in more valid or more precise GI values than the other methods tested here. The only method with an acceptable degree of agreement $(\leq \pm 5 \%)$ with the recommended method was AUCcut. These conclusions may not necessarily apply to the assessment of GI in subjects with diabetes.

\section{References}

Allison DB, Paultre F, Goran MI, Poehlman ET \& Heymsfield SB (1995) Statistical considerations regarding the use of ratios to adjust data. Int J Obes 9, 644-652.

Bland JM \& Altman DG (1986) Statistical methods for assessing agreement between two methods of clinical measurement. Lancet i, 307-310.

Brand Miller J, Foster-Powell K, Wolever TMS \& Colagiuri S (2002) The New Glucose Revolution: The Authoritative Guide to the Glycemic Index. New York: Marlowe \& Company.

Brand Miller JC, Hayne S, Petocz P \& Colagiuri S (2003) Lowglycemic index diets in the management of diabetes: a metaanalysis of randomized controlled trials. Diabetes Care 26, $2261-2267$.

Food and Agriculture Organization (1998) Carbohydrates in Human Nutrition. Report of an FAO/WHO Expert Consultation on Carbohydrates, April 14-18, 1997. Rome, Italy, Rome: FAO.

Frost G, Leeds A, Trew G, Margara R \& Dornhorst A (1998) Insulin sensitivity in women at risk of coronary heart disease and the effect of a low glycemic index diet. Metabolism 47, $1245-1251$.

Gannon MC \& Nuttall FQ (1987) Factors affecting interpretation of postprandial glucose and insulin areas. Diabetes Care 10, 759-763.

Gannon MC, Nuttall FQ, Westphal SA, Neil BJ \& Seaquist ER (1989) Effects of dose of ingested glucose on plasma metabolite and hormone responses in type II diabetic subjects. Diabetes Care 12, 544-552.

Ha M-A, Mann JI, Melton LD \& Lewis-Barned NJ (1992) Calculation of the glycaemic index. Diabetes Nutr Metab $\mathbf{5}$, $137-139$.

Jenkins DJA, Wolever TMS, Taylor RH, et al. (1981) Glycemic 
index of foods: a physiological basis for carbohydrate exchange. Am J Clin Nutr 34, 362-366.

Jenkins DJA, Wolever TMS, Kalmusky J, et al. (1987) Low-glycemic index diet in hyperlipidemia: use of traditional starchy foods. Am J Clin Nutr 46, 66-71.

Liu S, Willett WC, Stampfer MJ, et al. (2000) A prospective study of dietary glycemic load, carbohydrate intake and risk of coronary heart disease in US women. Am J Clin Nutr 71, 1455-1461.

Meyer KA, Kuski LTH, Jacobs DR, et al. (2000) Carbohydrates, dietary fiber, and incident type 2 diabetes in older women. Am J Clin Nutr 71, 921-930.

Salmeron J, Ascherio A, Rimm EB, et al. (1997a) Dietary fiber, glycemic load, and risk of NIDDM in men. Diabetes Care 20, 545-550.

Salmeron J, Manson JE, Stampfer MJ, et al. (1997b) Dietary fiber, glycemic load, and risk of non-insulin-dependent diabetes mellitus in women. J Am Med Assoc 277, 472-477.

Snedecor GW \& Cochran WB (1980) Statistical Methods, 7th ed. Ames, IA: Iowa State University Press.

Van Dam RM, Visscher AW, Feskens EJ, Verhoef P \& Kromhout
D (2000) Dietary glycemic index in relation to metabolic risk factors and incidence of coronary heart disease: the Zutphen Elderly Study. Eur J Clin Nutr 54, 726-731.

Vorster HH, Venter CS \& Silvis N (1990) The glycaemic index of foods: a critical evaluation. S Afr J Food Sci Nutr 1, 13-17.

Wolever TMS (1989) How important is prediction of glycemic responses? Diabetes Care 12, 591-593.

Wolever TMS (1992) Glycemic index vs glycemic response: nonsynonymous terms. Diabetes Care 15, 1436-1437.

Wolever TMS \& Jenkins DJA (1986) The use of the glycemic index in predicting the blood glucose response to mixed meals. Am J Clin Nutr 43, 167-172.

Wolever TMS, Jenkins DJA, et al. (1990) Glycemic index of foods in individual subjects. Diabetes Care 13, 126-132.

Wolever TMS \& Mehling C (2002) High-carbohydrate-low-glycaemic index dietary advice improves glucose disposition index in subjects with impaired glucose tolerance. Br J Nutr 87, 477-487.

Wolever TMS, Vorster HH, Björk I, et al. (2003) Determination of the glycaemic index of foods: interlaboratory study. Eur $J$ Clin Nutr 57, 475-482. 